Supporting Information for

\title{
Efficient Spin Selectivity in Self-Assembled Superhelical Conducting Polymer Microfibers
}

Lei Jia ${ }^{1,2, \dagger}$, Chenchen Wang ${ }^{1,3, \dagger}$, Yuchun Zhang ${ }^{1}$, Liu Yang ${ }^{4, *}$, and Yong Yan ${ }^{1,3, *}$

${ }^{1}$ CAS Key Laboratory of Nanosystem and Hierarchical Fabrication, CAS Center for Excellence in Nanoscience, National Center for Nanoscience and Technology, Beijing 100190, China

${ }^{2}$ School of Advanced Materials and Nanotechnology, Xidian University, Xi'an 710126, China

${ }^{3}$ University of Chinese Academy of Sciences, Beijing, 100049, China

${ }^{4}$ School of Physics and Electronic Engineering, Linyi University, Linyi, 276005, China

- section S1. Additional experimental results

- S1.1. Structural analysis of Superhelical PANI microfiber

- Figure S1. Characterization of superhelical PANI microfibers.

- S1.2. Asymmetric electrodes fabrication, statistics of channel length, and contact issue

- Figure S2. The procedure for fabrication of asymmetric electrodes device.

- Figure S3. The statistics of channel length.

- Figure S4. The contact between electrodes and PANI.

- S1.3. Current-voltage (I-V) characteristics of the lateral devices

- Figure S5. Current-voltage (I-V) characteristics at the absence of magnetic field.

- Figure S6. Current-voltage (I-V) characteristics of Left-PANI with parallel magnetic field.

- Figure S7. Current-voltage (I-V) characteristics of Left-PANI with antiparallel magnetic field.

- Figure S8. Current-voltage (I-V) characteristics of Right-PANI with parallel magnetic field.

- Figure S9. Current-voltage (I-V) characteristics of Right-PANI with antiparallel magnetic field.

- S1.4. Procedure to plot the $I-V$ and $\mathrm{dI} / \mathrm{dV}$ curves

- S1.5. Six additional superhelical PANI microfiber devices

- Figure S10. Three additional left-PANI microfiber devices.

- Figure S11. Three additional right-PANI microfiber devices.

- S1.6. Control devices-Au/Chiral-PANI/Au

- Figure S12. Au/Chiral-PANI/Au devices.

- S1.7. Control device-Ni/Achiral-PANI/Au 
- Figure S13. Ni/Achiral-PANI/Au device.

- S1.8. Antisymmetric magnetoresistance

- Figure S14. Antisymmetric magnetoresistance curves

- S1.9. Efficient spin selectivity of eight devices

- Figure S15. Spin selectivity.

- S1.10. Magnetic property of Ni electrode

- Figure S16. The magnetic hysteresis loop.

- S1.11. Simulation parameters

- Figure S17. TEM images of superhelical PANI microfibers.

- table S1. The parameters of individual microfibers and the average data

- table S2. The parameters used in the model

- section S2. Theory details

- Figure S18. Simulation of spin selectivity in helical PANI microfibers.

- References

\section{section S1. Additional experimental results}

\section{S1.1. Structural analysis of Superhelical PANI microfiber}

Figure S1 showed the characterization results of superhelical PANI microfibers. The SEM (Figure S1a-c) and TEM (Figure S1e-f) images displayed clear helical screws. The chiral PANI molecules arranged orderly in these superhelical fibers which was confirmed by using selected area electron diffraction (Figure S1e-f inset) and X-ray diffraction (Figure S1g) techniques. Besides the $\pi-\pi$ stacking of chiral PANI molecules (040, $d$ spacing of $c a .3 .5 \AA$ ), the sharp reflections associated with a $d$ spacing of $c a$. $6.1 \AA$ from the (100) reflection are due to the arrangement of CSA molecules from the neighboring PANI chains. The strongest peak in XRD pattern with a $d$ spacing of $c a$. $28 \AA$, ascribed to (001) reflection, was overwhelmed by the intense transmission beam in the SAED pattern. However, a sharp reflection corresponding to a $d$ spacing of $c a$. $10 \AA$ could probably come from the (003) reflection of the same diffraction family. 

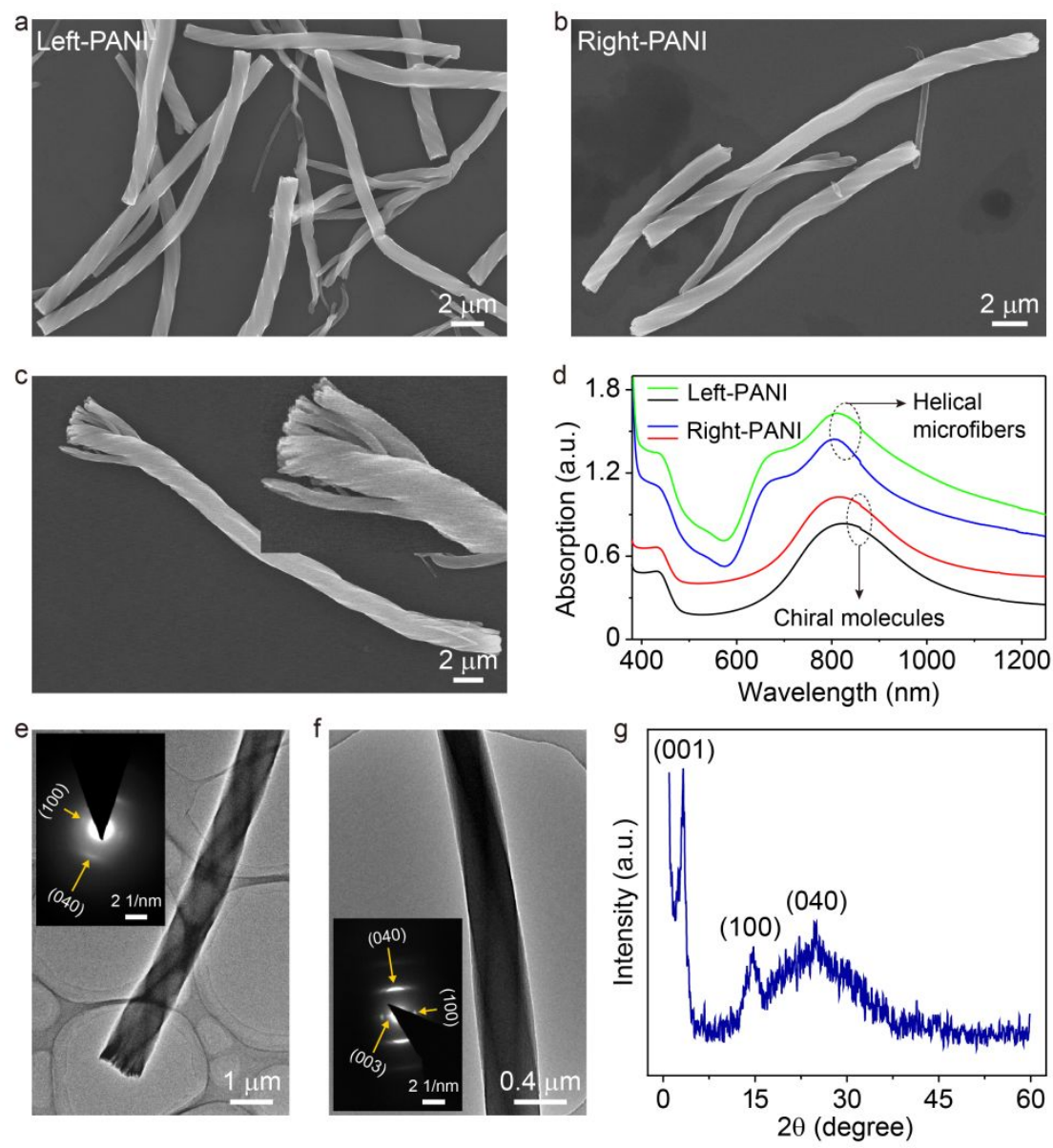

Figure S1. Characterization of superhelical PANI microfibers. a) and b) SEM images of left- and right-handed helical PANI microfibers induced by using $D$ - and $L$-CSA dopant acids. c) A SEM image showing the superhelical microfibers were formed by many helical nanofibers which twisted along the same screw direction of microfibers. d) The UV-vis-IR spectra of chiral PANI molecules and helical microfibers. e) and f) The TEM images and corresponding SAED pattern (inset) of helical PANI microfiber and nanofiber respectively. g) XRD spectrum of helical PANI microfibers. 
S1.2. Asymmetric electrodes fabrication, statistics of channel length, and contact issue.
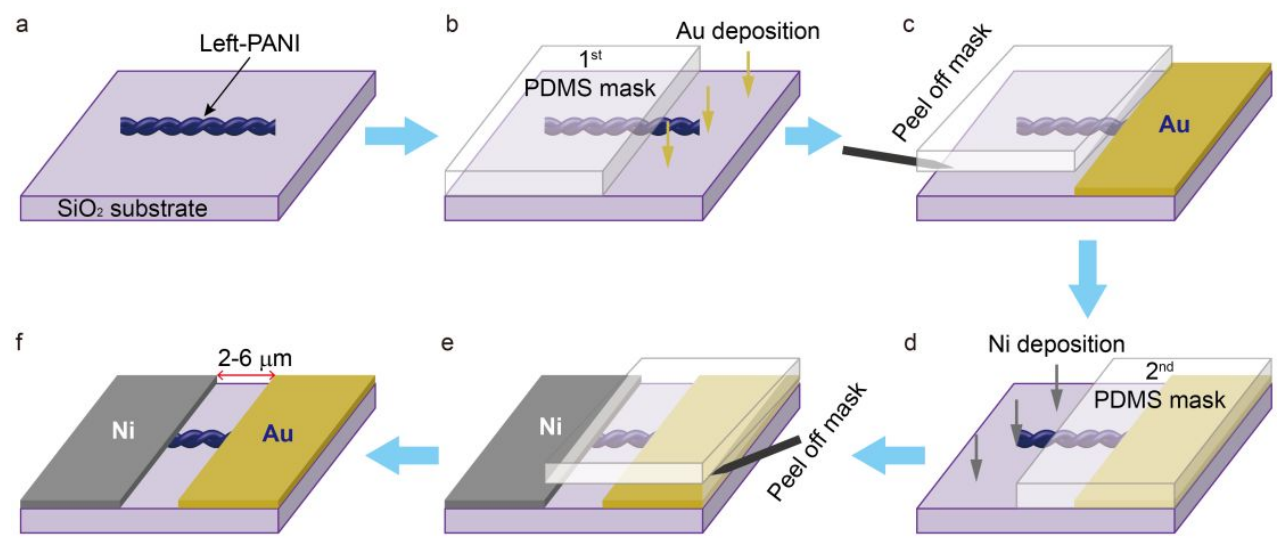

d Ni deposition

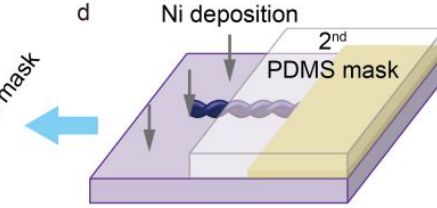

Figure S2. The procedure for fabrication of asymmetric electrodes device. a) PANI microfibers was firstly drop-casted on silica substrate where an individual fiber could be identified by the assistance of an optical microscopy (X600 magnification). b) A PDMS mask ( $c a .5 \mu \mathrm{m}$ in thick) was placed to partly cover the helical microfiber, followed by Au electrode deposition (400 nm, e-beam evaporation). c) The PDMS was peeled off with the help of a probe. d) Another PDMS mask was placed to cover the $\mathrm{Au}$ electrode and the "channel". Ni electrode (400 nm) was subsequently deposited by using e-beam evaporation. e) The second PDMS mask was peeled off. f) A two-terminal lateral device with asymmetric electrodes was produced where the channel length was in the range of 2-6 $\mu \mathrm{m}$. 


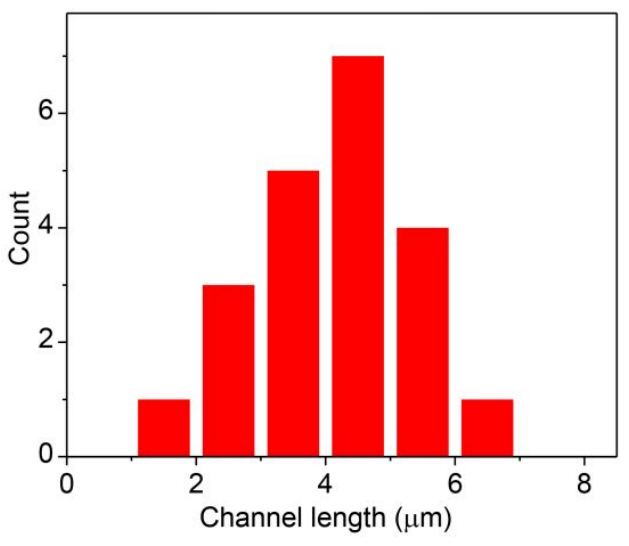

Figure S3. The statistics of channel length. The channel length of two-terminal lateral devices with asymmetric electrodes was in the range of 2-6 $\mu \mathrm{m}$. 
The contact between metallic electrodes and PANI microfiber was characterized by performing local potential measurements under the assistance of Kelvin force microscopy (KFM, Bruker Multimode 8-HR). The measurement results were shown in Figure S4. To study the potential distributions along the device, four points (A, B, C, and D) were selected. The potential drop along the microfiber was steady (between B and $\mathrm{C}$ ). There was negligible local potential drop around the Au-PANI junction (between $\mathrm{C}$ and D), demonstrating its Ohmic contact characteristics. However, significant potential drop was found at Ni-PANI junction (between A and B), indicating this barrier was highly resistive and crucial for electron transport at both negative and positive biases. In addition, no current rectifying characteristics were observed for these asymmetric electrode devices. Therefore, the Ni-PANI junction could be regarded as a tunnel junction rather than a Schottky barrier. It is known that Ni is prone to oxidize at ambient condition, we surmised that this resistive barrier was due to the formation of Nickel oxide layer.
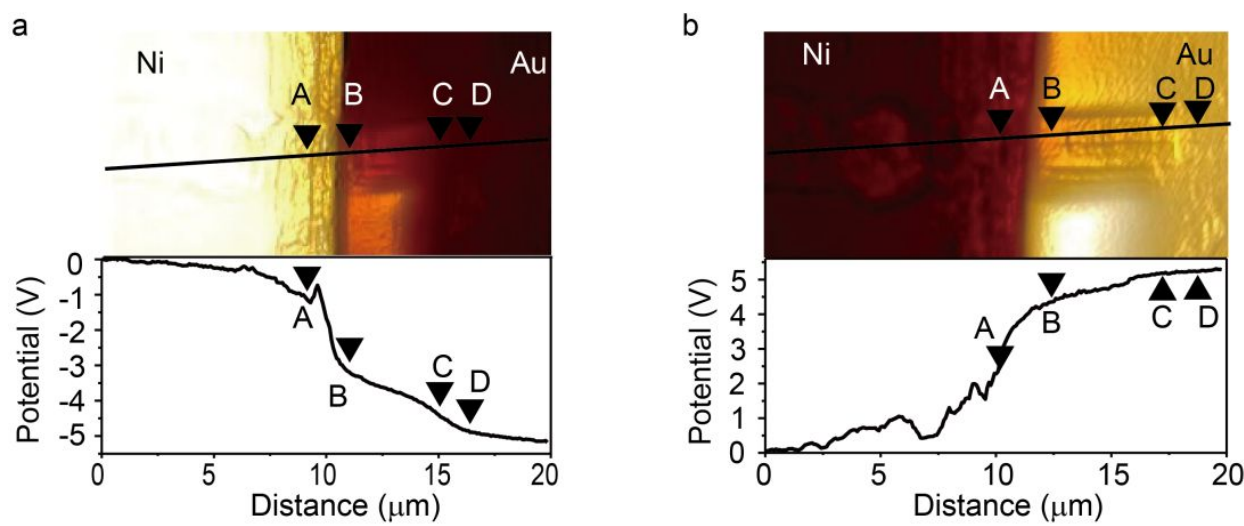

Figure S4. The contact between electrodes and PANI. Surface potential images and profiles of a typical PANI microfiber device with asymmetric Au and Ni electrodes at biases of (a) - $5 \mathrm{~V}$ and (b) $5 \mathrm{~V}$. 


\section{S1.3. Current-voltage $(I-V)$ characteristics of the lateral devices}

To confirm the reliability or our measurements, the $I-V$ was scanned for ten cycles at each condition, with parallel, antiparallel, and absence of magnetic field, no hysteresis or distinct deviation has been detected. The magnetic field was step-increased from 0 (absence of field) to 10,000 Oe and the average $I-V$ of 10 curves was calculated. This section is the raw data used to plot Figure $2 \mathrm{a}$ and $2 \mathrm{~b}$.
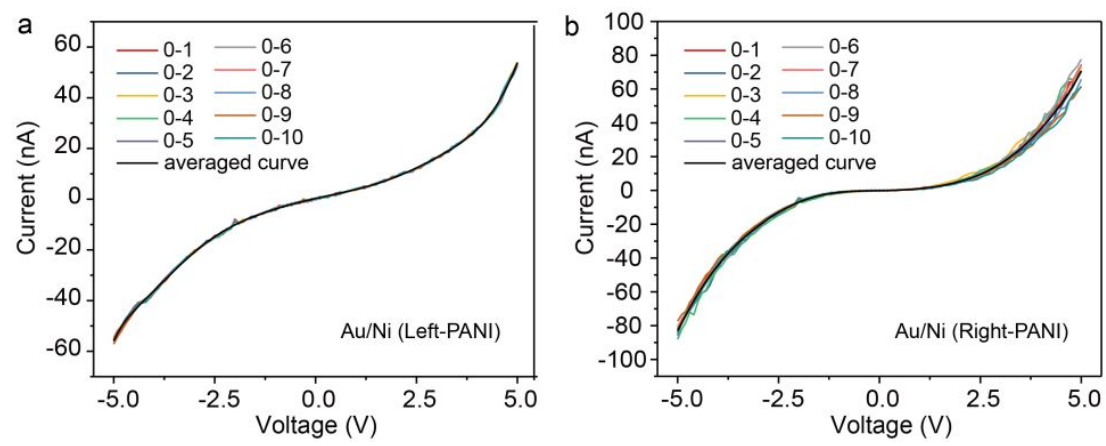

Figure S5. Current-voltage $(I-V)$ characteristics at the absence of magnetic field. The raw data of a) left- and b) right-handed helical PANI microfiber devices. Ten cycles were recorded, and the average $I-V$ curve was plotted in black. All the measurements were performed at $300 \mathrm{~K}$. 

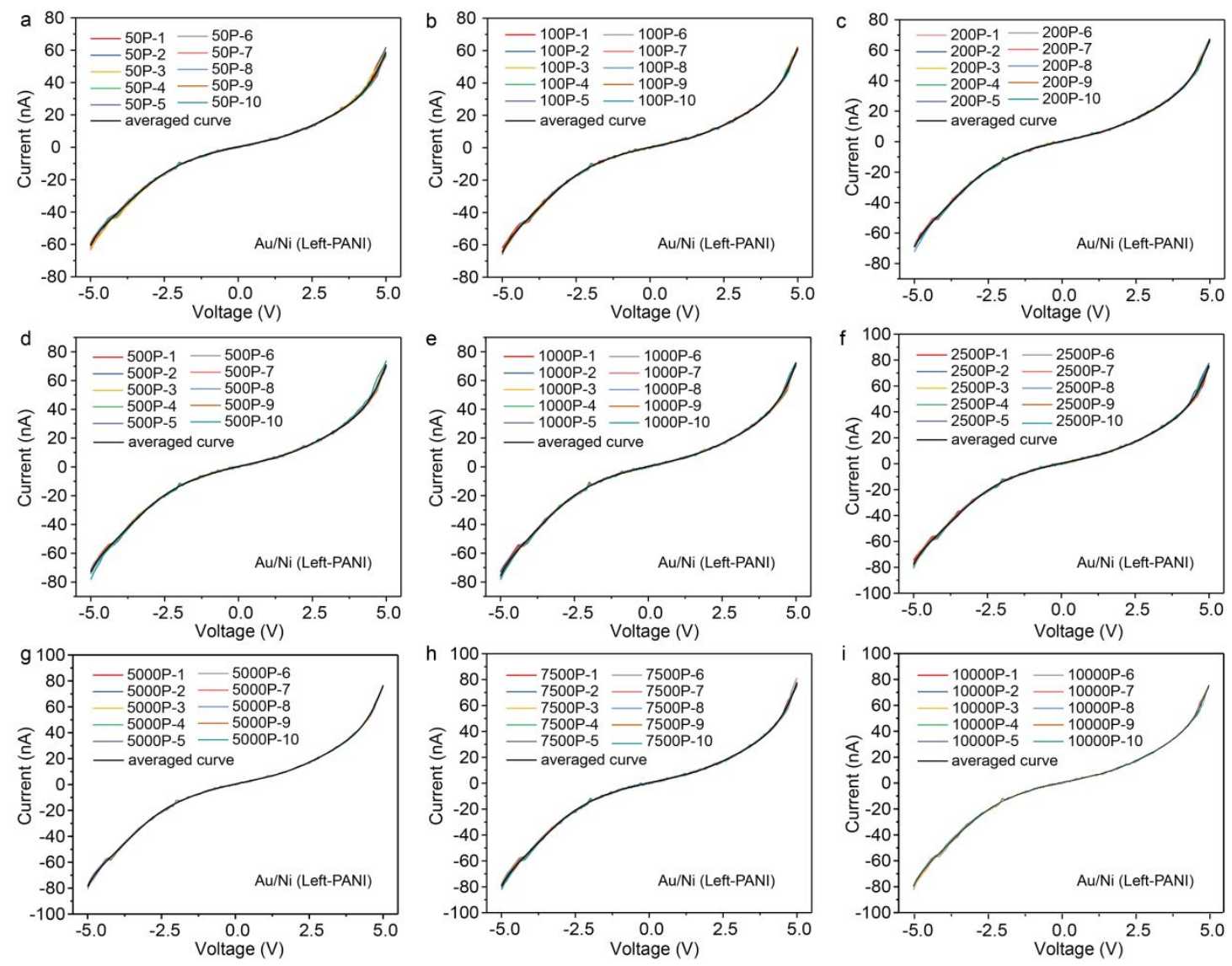

Figure S6. Current-voltage $(I-V)$ characteristics of Left-PANI with parallel magnetic field. The raw data (ten cycles) and average (black curve) $I-V$ curves of the left-handed helical PANI device at the magnetic field increased from 50 to 10,000 Oe. All the measurements were performed at $300 \mathrm{~K}$. 

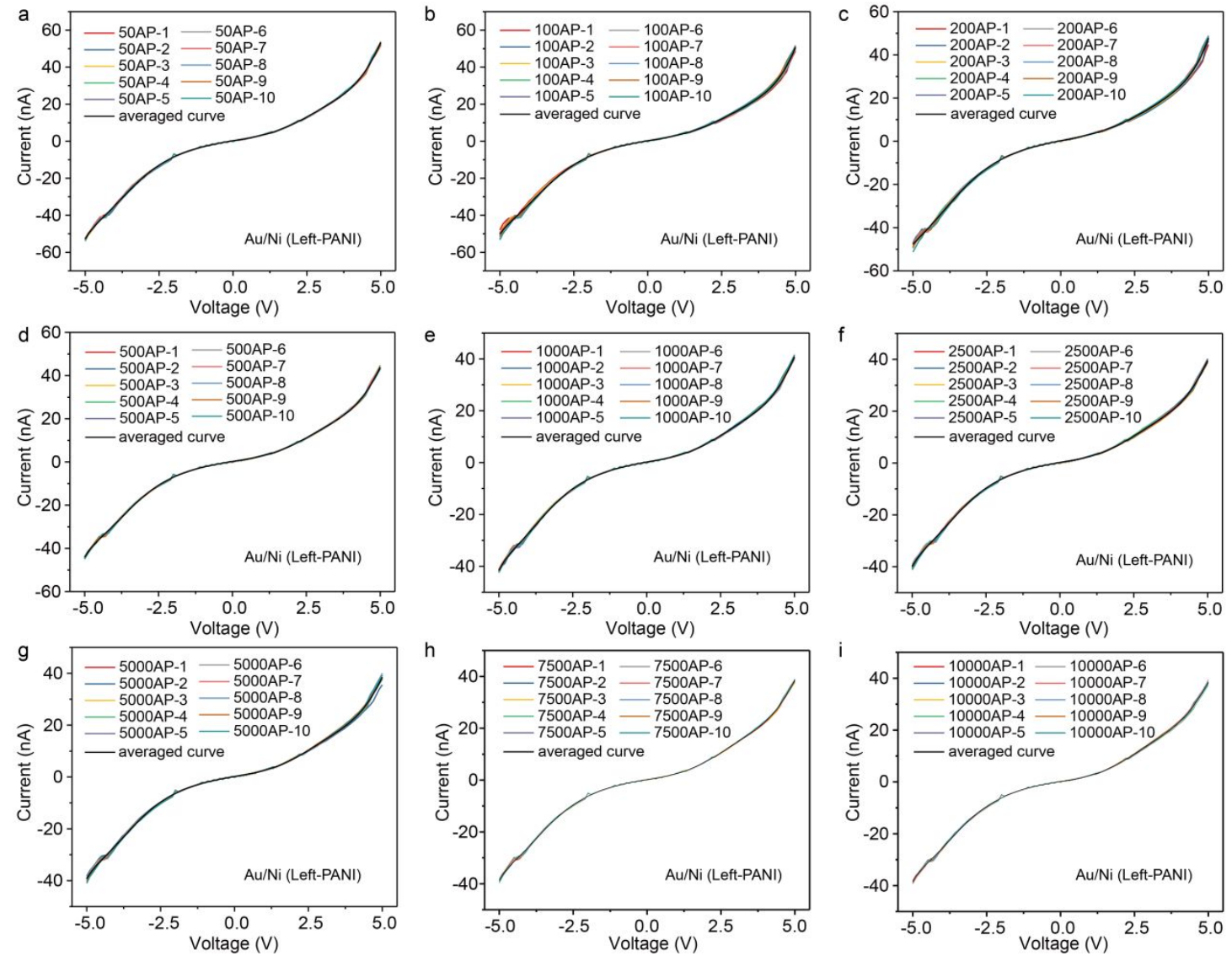

Figure S7. Current-voltage $(I-V)$ characteristics of Left-PANI with antiparallel magnetic field. The raw data (ten cycles) and average (black curve) $I-V$ curves of the left-handed helical PANI device at the magnetic field increased from 50 to 10,000 Oe. All the measurements were performed at $300 \mathrm{~K}$. 

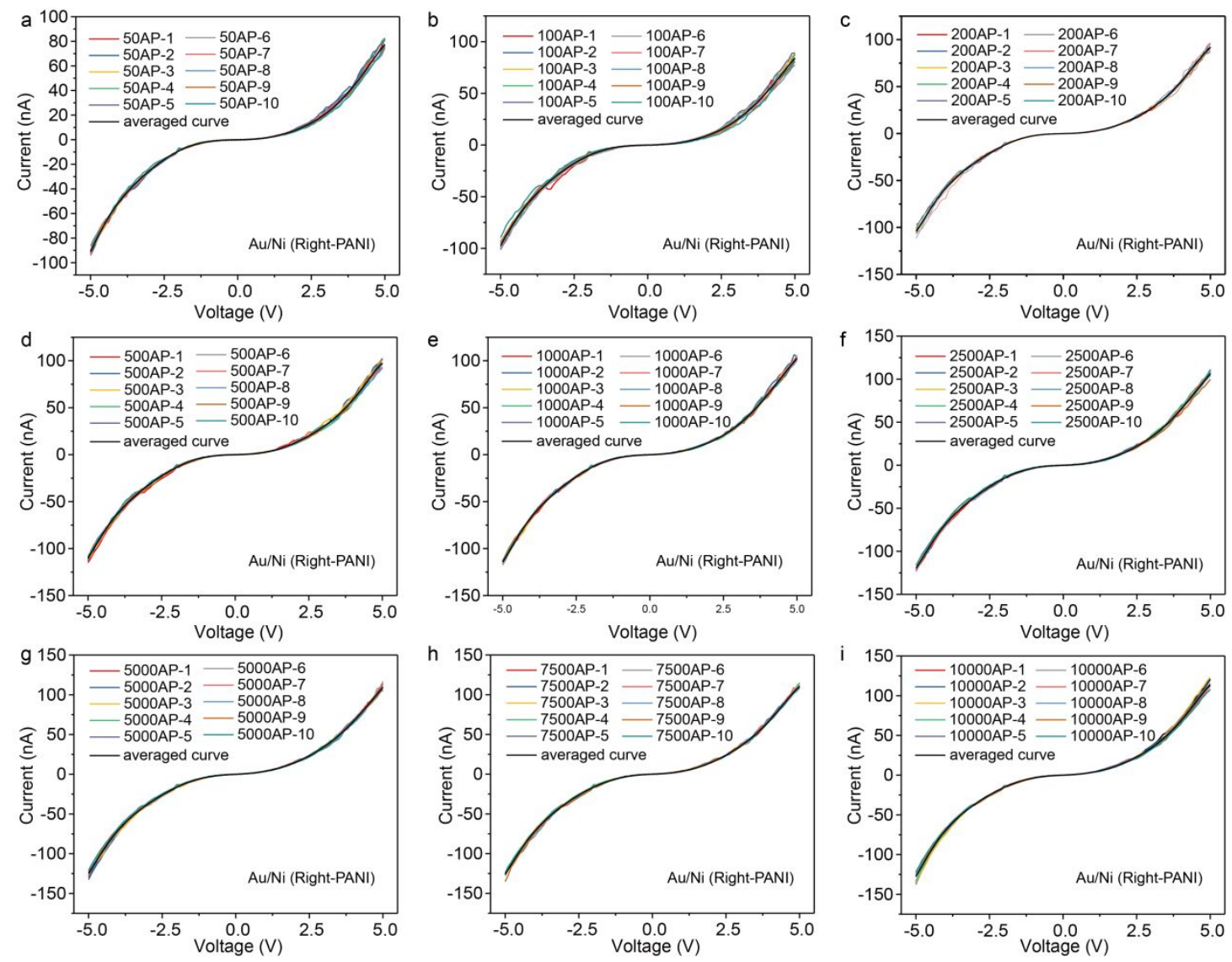

Figure S8. Current-voltage $(I-V)$ characteristics of Right-PANI with parallel magnetic field. The raw data (ten cycles) and average (black curve) $I-V$ curves of the right-handed helical PANI device at the magnetic field increased from 50 to 10,000 Oe. All the measurements were performed at $300 \mathrm{~K}$. 

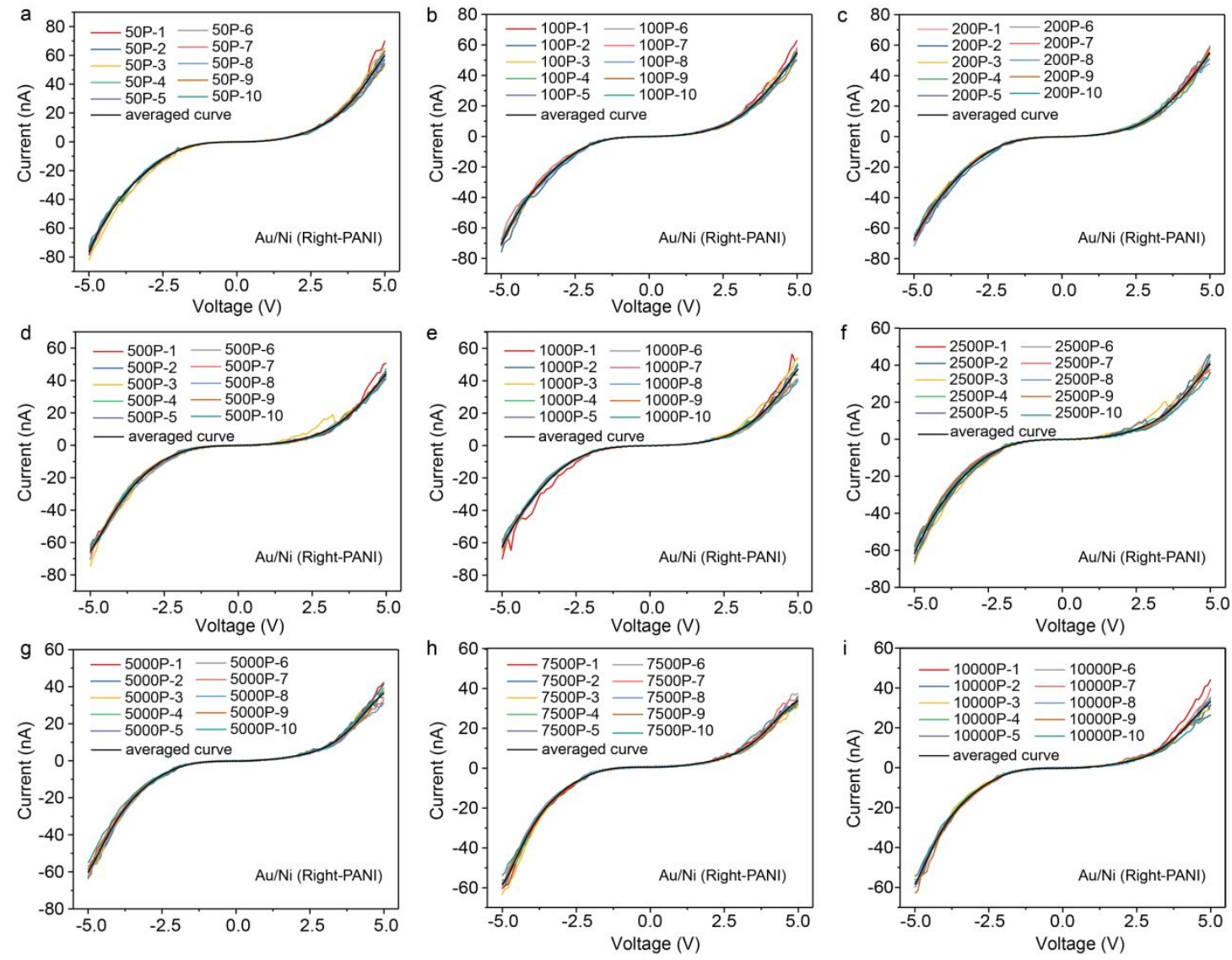

Figure S9. Current-voltage $(I-V)$ characteristics of Right-PANI with antiparallel magnetic field. The raw data (ten cycles) and average (black curve) $I-V$ curves of the right-handed helical PANI device at the magnetic field increased from 50 to 10,000 Oe. All the measurements were performed at $300 \mathrm{~K}$. 


\section{S1.4. Procedure to plot the $I-V$ and $d I / d V$ curves}

The detailed procedure to plot the $I-V$ and $\mathrm{dI} / \mathrm{dV}$ curves in the main text and Supporting information: $I-V$ curves, the current-voltage measurement was performed by scanning bias from $-5 \mathrm{~V}$ to $+5 \mathrm{~V}$ with a bias step increase of $100 \mathrm{mV}$. Total of 101 scattering data points was collected and plotted into a continuous line shape of one $I-V$ curve. Next, the average of $10 I-V$ curves (the bias scanning was repeated for ten times) was plotted into Figure 2a-c, Figure S10a, c, e, Figure S11a, c, e, Figure S12c-f, and Figure $\mathrm{S} 13 \mathrm{~b}$, c. It must note that the current value at $\mathrm{V}=0 \mathrm{~V}$ is a random data, not an effective data point. In other words, there was no effective data between $-100 \mathrm{mV}$ and $+100 \mathrm{mV} ; \mathrm{dI} / \mathrm{dV}$ curves, in software OriginPro 2017, the 101 scattering data points was subjected to perform first derivative differentiate operation. The detailed steps were, selected the data column $\rightarrow$ Analysis $\rightarrow$ Mathematics $\rightarrow$ Differentiate $\rightarrow$ Select differentiate order 1. By this way, we plotted Figure 2-2f, Figure S10b, d, f, Figure S11b, d, f, Figure S12g, h, and Figure S13d. Again, the dI/dV curve contained 101 scattering data points and there was no effective data points between $-100 \mathrm{mV}$ and $+100 \mathrm{mV}$. 


\section{S1.5. Six additional superhelical PANI microfiber devices}
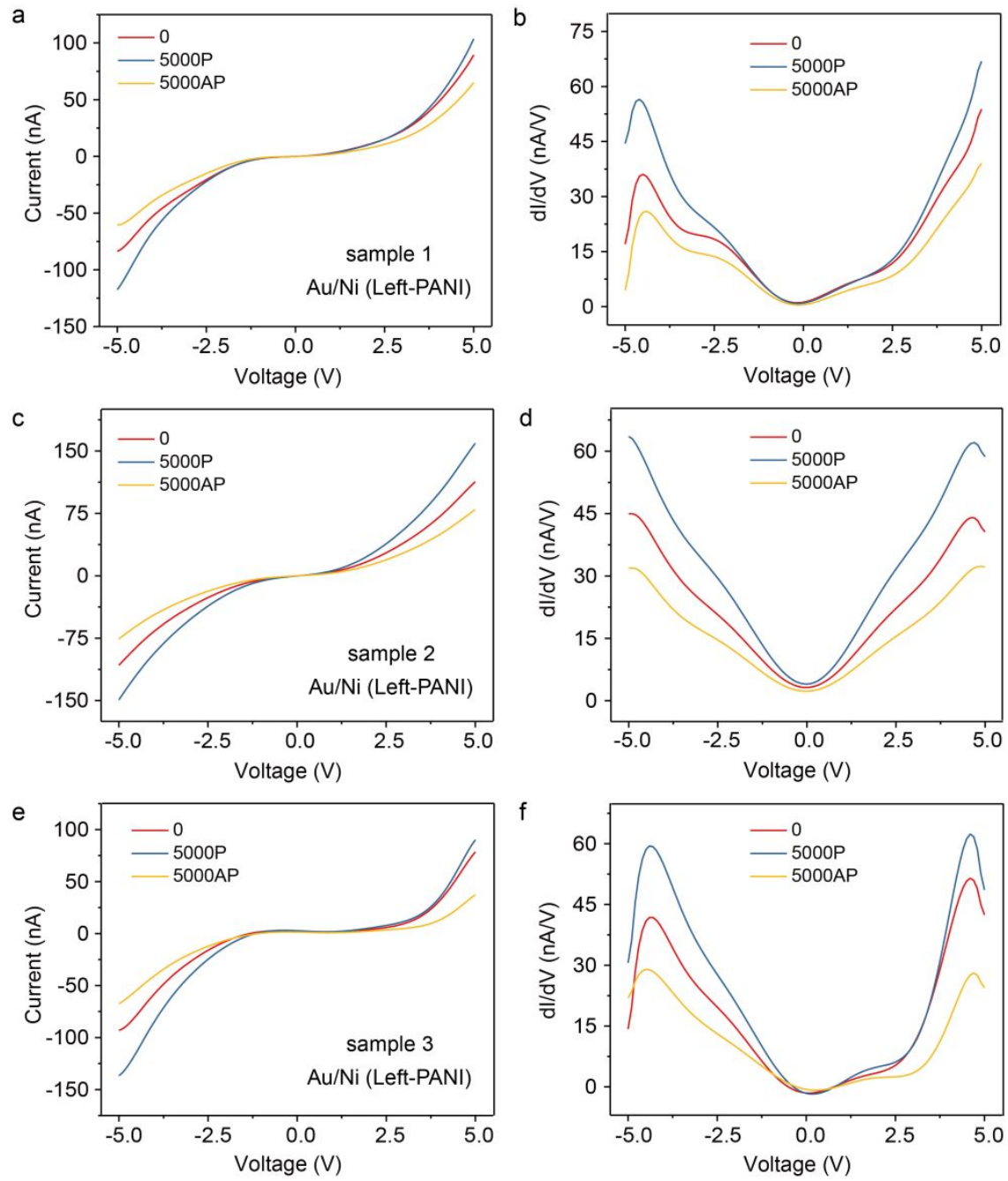

Figure S10. Three additional left-PANI microfiber devices. Current-voltage $(I-V)$ characteristics and density of states of three additional left-handed helical PANI microfibers devices with parallel, antiparallel, and absence of magnetic field (5, 000 Oe). All the measurements were performed at $300 \mathrm{~K}$. 

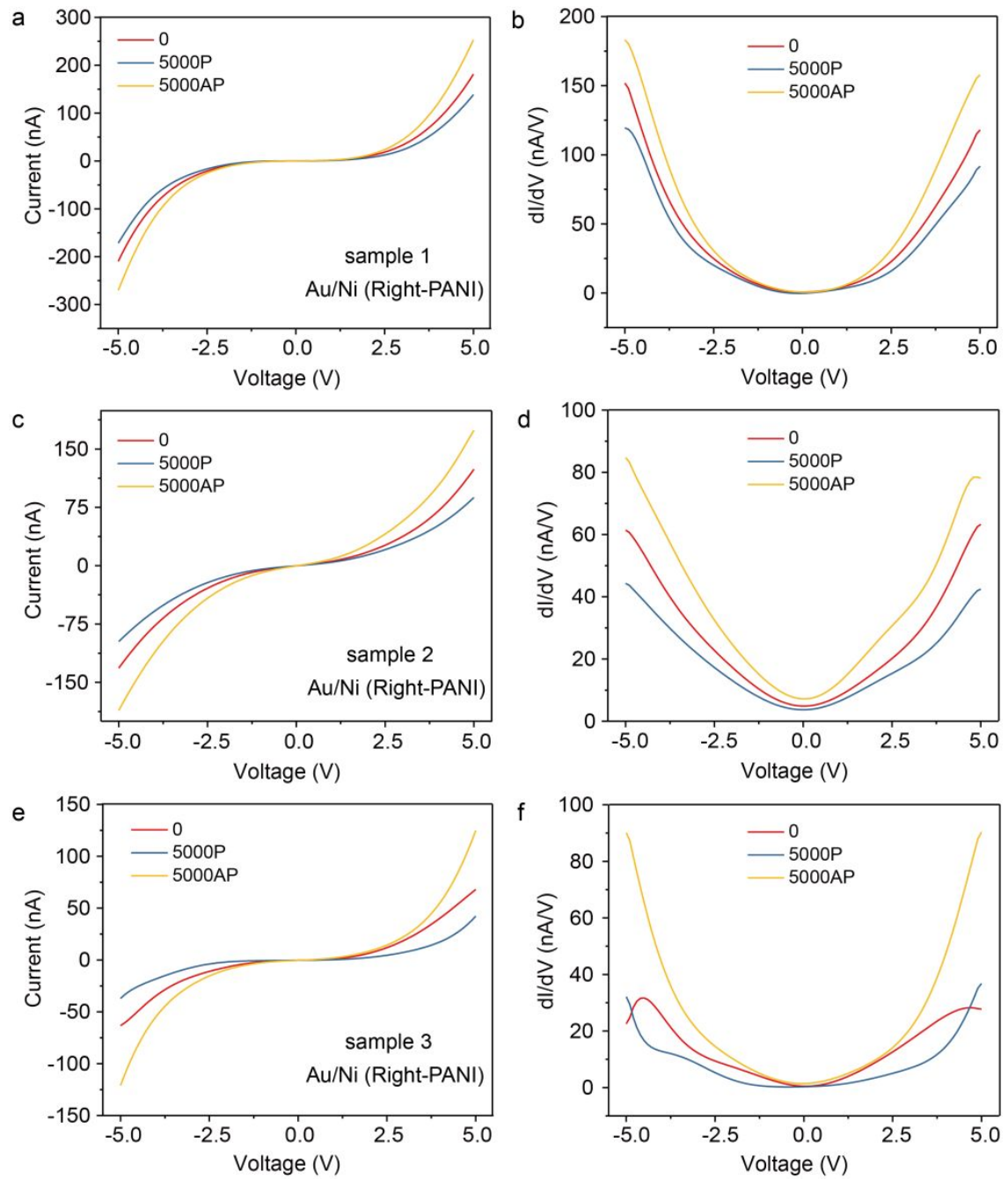

Figure S11. Three additional right-PANI microfiber devices. Current-voltage $(I-V)$ characteristics and density of states of three additional right-handed helical PANI microfibers devices with parallel, antiparallel, and absence of magnetic field $(5,000$ Oe). All the measurements were performed at $300 \mathrm{~K}$. 


\section{S1.6. Control devices-Au/Chiral-PANI/Au}
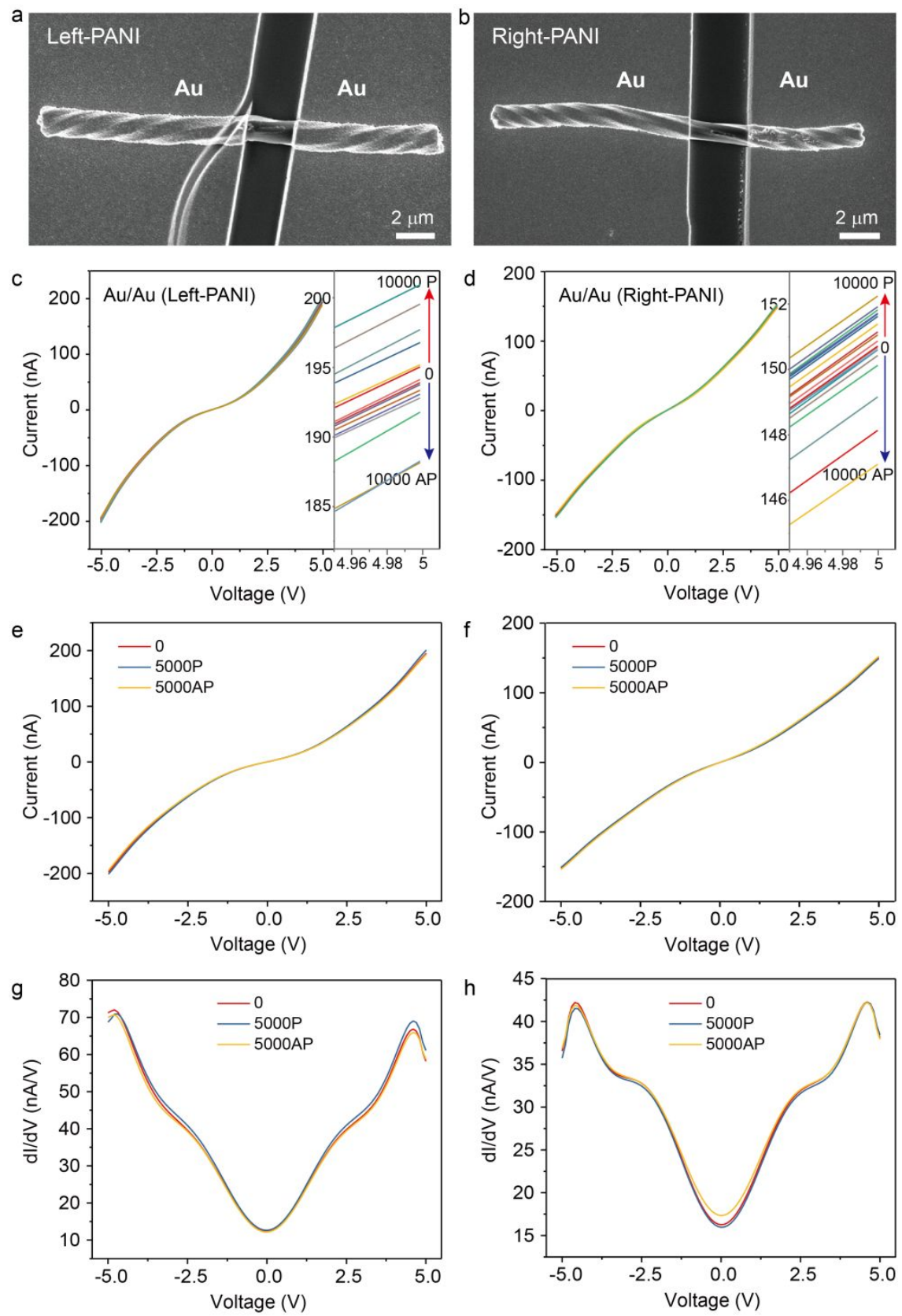

Figure S12. Au/Chiral-PANI/Au devices. a) and b) Typical SEM images, c) and d) current-voltage characteristics, of the $\mathrm{Au} / \mathrm{Chiral-PANI} / \mathrm{Au}$ lateral devices with left- and right-handed PANI helical microfibers respectively. e) and f) I-V curves, g) and h) $\mathrm{dI} / \mathrm{dV} \sim \mathrm{V}$ for left- and right-handed helical PANI microfibers with parallel, antiparallel, and absence of magnetic field (5,000 Oe). No distinct spin selective characteristics were observed for these devices. All the measurements were performed at $300 \mathrm{~K}$. 


\section{S1.7. Control device-Ni/Achiral-PANI/Au}
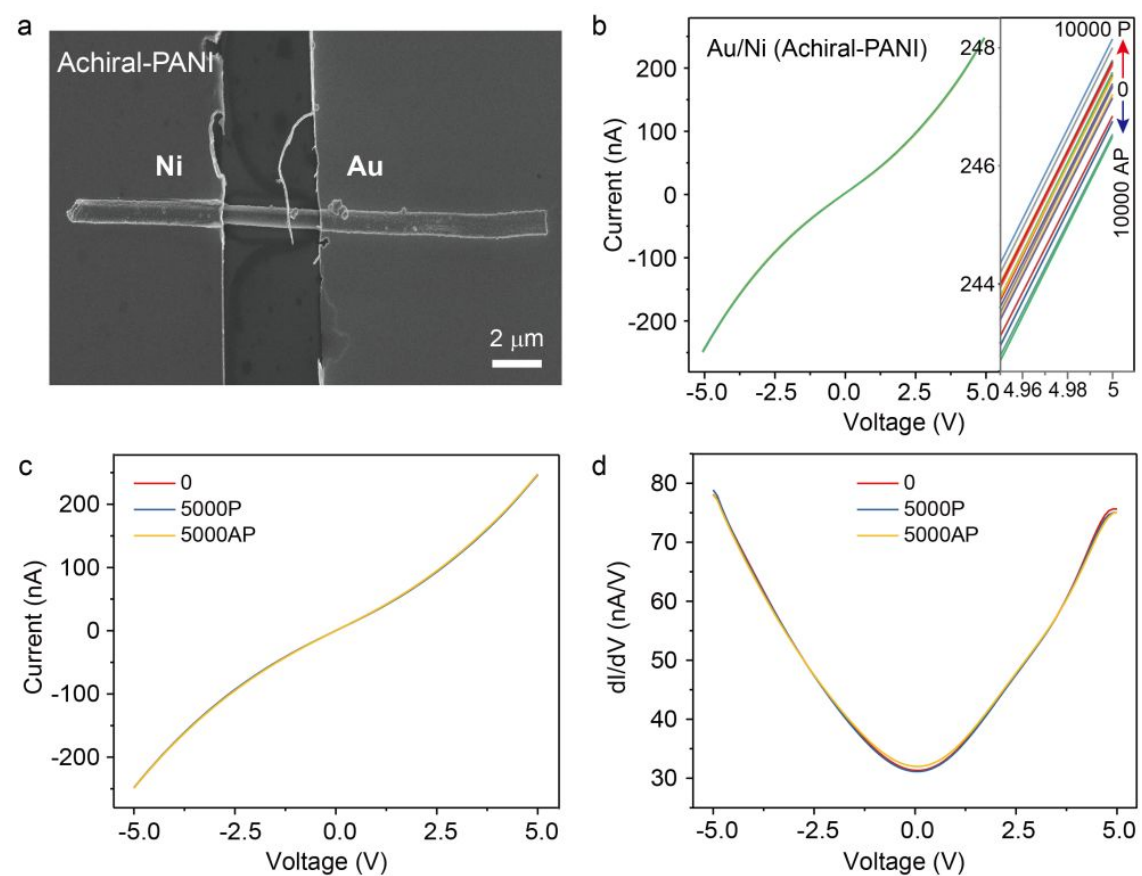

Figure S13. Ni/Achiral-PANI/Au device. a) and b) Typical SEM image and currentvoltage characteristics of the Ni/Achiral-PANI/Au lateral device respectively. c) and d) I-V curves and $\mathrm{dI} / \mathrm{dV} \sim \mathrm{V}$ for the achiral PANI microfiber with parallel, antiparallel, and absence of magnetic field $(5,000 \mathrm{Oe})$. No distinct spin selective characteristic was observed for this device. The measurements were performed at $300 \mathrm{~K}$. 


\section{S1.8. Antisymmetric magnetoresistance}
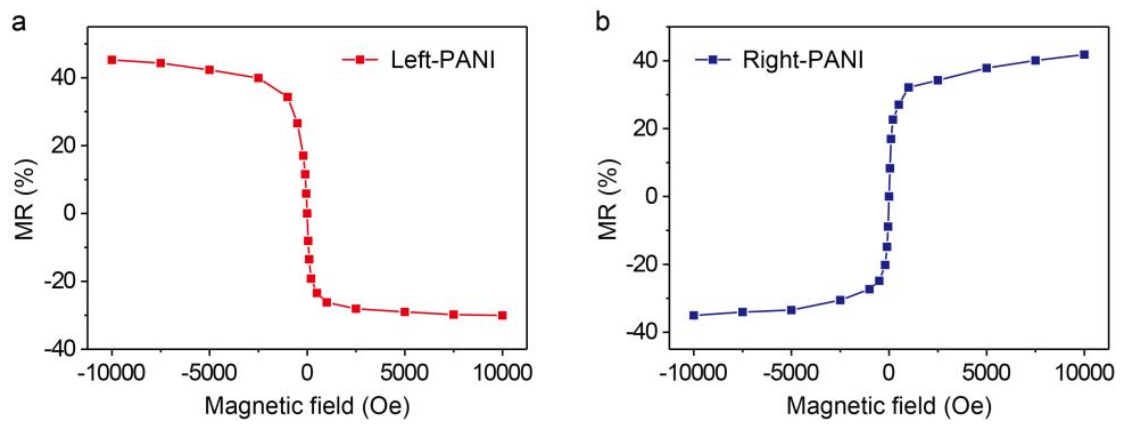

Figure S14. Antisymmetric magnetoresistance curves. The resistance of left- and righthanded helical PANI microfibers as function of magnetic field. The MRs were calculated by placing $5 \mathrm{~V}$ on the Ni/chiral-PANI/Au lateral devices. The measurements were performed at $300 \mathrm{~K}$.

\section{S1.9. Efficient spin selectivity of eight devices}

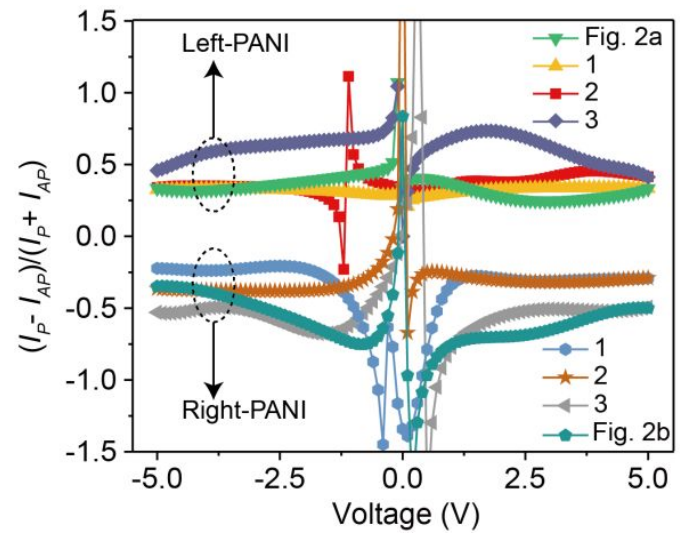

Figure S15. Spin selectivity. The spin selectivity as a function of placed bias for leftand right-handed helical PANI microfibers in the lateral devices of $\mathrm{Ni} / \mathrm{Chiral}$ PANIs/Au. Four left- and four right-handed PANI devices were plotted. The magnetic field is 5,000 Oe. 


\section{S1.10. Magnetic property of Ni electrode}

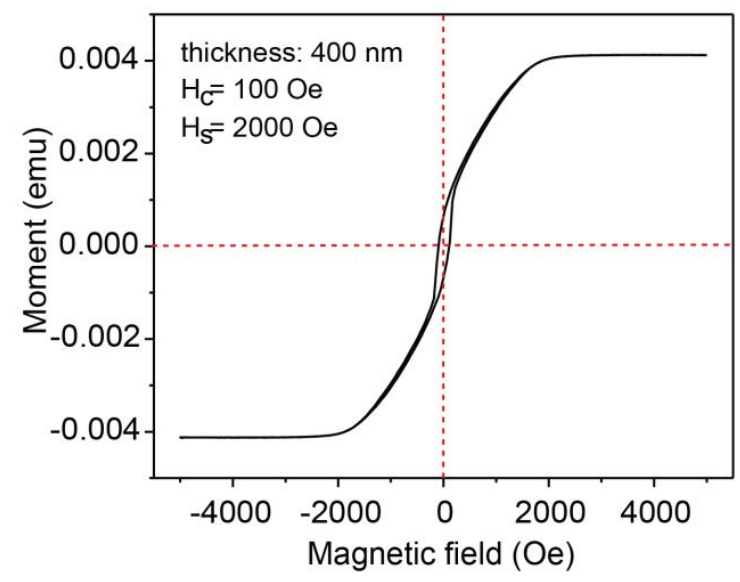

Figure S16. The magnetic hysteresis loop. The magnetic property of Ni electrode was characterized by using PPMS, where $H_{C} \sim 100$ 0e and $H_{S} \sim 2,000$ Oe were detected. The thickness of Ni electrode is $400 \mathrm{~nm}$. The measurement was performed at $300 \mathrm{~K}$.

\section{S1.11. Simulation parameters}
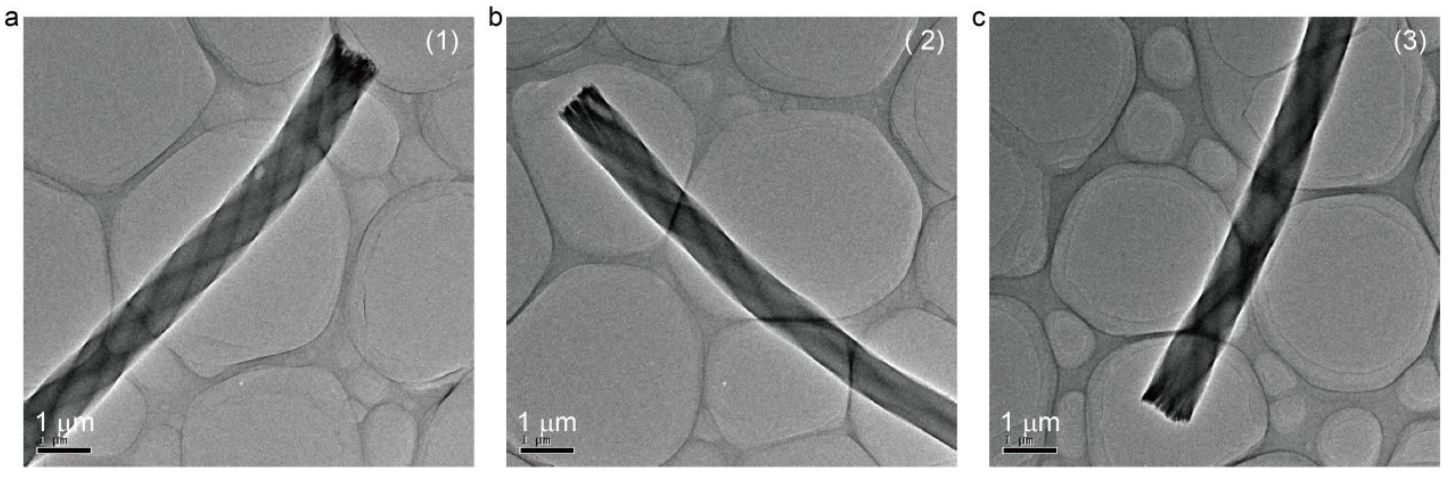

Figure S17. TEM images of superhelical PANI microfibers. Three microfibers were used to calculate the helical pitch, twist angle $(\Delta \varphi)$, and helix angle $(\phi)$. 
Table S1. The parameters of individual microfibers and the average data

\begin{tabular}{c|c|c|c|c}
\hline NO. & Diameter (nm) & Helical pitch (nm) & $\Delta \boldsymbol{\varphi} \mathbf{(}^{\mathbf{0}}$ & $\left.\boldsymbol{\phi} \mathbf{(}^{\mathbf{0}}\right)$ \\
\hline 1 & 987.6 & 4540.1 & 0.028 & 55.6 \\
\hline 2 & 835.8 & 4961.4 & 0.025 & 62.1 \\
\hline 3 & 911.9 & 5172.0 & 0.024 & 61.0 \\
\hline Average & $\mathbf{9 1 1 . 8}$ & $\mathbf{4 8 9 1 . 2}$ & $\mathbf{0 . 0 2 6}$ & $\mathbf{5 9 . 6}$ \\
\hline
\end{tabular}

Table S2. The parameters used in the model

\begin{tabular}{ccccccccccc}
\hline parameter & $\mathbf{t}_{\mathbf{0}}$ & $\boldsymbol{\alpha}$ & $\mathbf{K}$ & $\boldsymbol{M}$ & $\boldsymbol{a}$ & $\boldsymbol{t}_{\text {so }}$ & $\boldsymbol{U}$ & $\boldsymbol{E}$ & $\Delta \boldsymbol{\varphi}$ & $\boldsymbol{\phi}$ \\
\hline value & 2.5 & 4.1 & 21 & 1349.14 & 1.22 & 0.125 & 0.5 & 0.25 & 0.03 & 60 \\
unit & $\mathrm{eV}$ & $e V / \AA$ & $e V / \AA^{2}$ & $\begin{array}{c}e V \cdot f s^{2} \\
/ \AA^{2}\end{array}$ & $\AA$ & $e V$ & $e V$ & $m V / \AA$ & $\circ$ & $\circ$ \\
\hline
\end{tabular}




\section{section S2. Theory details}

We consider the transmission of electrons in helical PANI microfibers as a quasi-onedimensional hopping mechanism, and the Hamiltonian could be described by using an extended tight-binding Su-Schrieffer-Heeger (SSH) model where the Rashba spin-orbit coupling (SOC) and Hubbard electron-electron interaction are considered ${ }^{1-3}$,

$$
\mathrm{H}=H_{S S H}+H_{S O C}+H_{e e}+H_{E}
$$

The first term represents the Hamiltonian of SSH model,

$$
\begin{gathered}
H_{S S H}=-\sum_{n, S}\left[t_{0}-\alpha\left(u_{n+1}-u_{n}\right)\right]\left(C_{n, S}^{+} C_{n+1, s}+C_{n+1, S}^{+} C_{n, S}\right)+\frac{1}{2} K \\
\sum_{n}\left(u_{n+1}-u_{n}\right)^{2}+\frac{1}{2} M \sum_{n} \dot{u}_{n}^{2}
\end{gathered}
$$

Where $t_{0}$ is the electron transfer integral in a uniform lattice, $\alpha$ is the electron-lattice coupling parameter, and $u_{n}$ is the lattice displacement at the $n^{\text {th }}$ site. $C_{n, s}^{+}\left(C_{n, s}\right)$ is the electron creation (annihilation) operator of $\pi$ electrons at $n^{\text {th }}$ site with spin $s . K$ denotes the elastic constant, and $M$ is the mass of a monomer. The second term in Eq. (1) is the Rashba spin-orbit coupling ${ }^{4}$,

$$
H_{S O C}=\sum_{n, s, S^{\prime}} 2 i t_{s o} \cos \varphi_{n}^{-} C_{n, s}^{+} \sigma_{n S S^{\prime}} C_{n+1, S^{\prime}}+\text { h.c. }
$$

where $t_{s o}$ donates spin-orbit coupling strength, $\varphi_{n}^{ \pm}=\left(\varphi_{n+1} \pm \varphi_{n}\right) / 2, \varphi_{n}=n \Delta \varphi$, and $\Delta \varphi$ is the twist angle between the neighboring $\pi$ planes. $\sigma_{n s S^{\prime}}=$ $\left(\sigma_{s s^{\prime}}^{x} \sin \varphi_{n}^{+}-\sigma_{s s^{\prime}}^{y} \cos \varphi_{n}^{+}\right) \sin \phi+\sigma_{s S^{\prime}}^{z} \cos \phi$, where $\sigma_{s S^{\prime}}^{x}, \sigma_{S S^{\prime}}^{y}$ and $\sigma_{s S^{\prime}}^{z}$ is the Pauli matrix, and $\phi$ is the helix angle. The third term is the Hubbard electron-electron interaction ${ }^{5}$ which is important in electron transportation ${ }^{6-7}$,

$$
H_{e e}=\sum_{n} U C_{n, \uparrow}^{+} C_{n, \uparrow} C_{n, \downarrow}^{+} C_{n, \downarrow}
$$

where $U$ donates electron-electron interaction strength, and we treat the e-e interaction with mean field approximation as, 


$$
\begin{gathered}
\mathrm{C}_{n \uparrow}^{+} C_{n \uparrow} \mathrm{C}_{n \downarrow}^{+} C_{n \downarrow} \approx\left[\left\langle\mathrm{C}_{n \uparrow}^{+} C_{n \uparrow}\right\rangle \mathrm{C}_{n \downarrow}^{+} C_{n \downarrow}+\left\langle\mathrm{C}_{n \downarrow}^{+} C_{n \downarrow}\right\rangle \mathrm{C}_{n \uparrow}^{+} C_{n \uparrow}-\left\langle\mathrm{C}_{n \uparrow}^{+} C_{n \uparrow}\right\rangle\left\langle\mathrm{C}_{n \downarrow}^{+} C_{n \downarrow}\right\rangle\right]- \\
{\left[\left\langle\mathrm{C}_{n \uparrow}^{+} C_{n \downarrow}\right\rangle \mathrm{C}_{n \downarrow}^{+} C_{n \uparrow}+\left\langle\mathrm{C}_{n \downarrow}^{+} C_{n \uparrow}\right\rangle \mathrm{C}_{n \uparrow}^{+} C_{n \downarrow}-\left\langle\mathrm{C}_{n \downarrow}^{+} C_{n \uparrow}\right\rangle\left\langle\mathrm{C}_{n \uparrow}^{+} C_{n \downarrow}\right\rangle\right]}
\end{gathered}
$$

An electric field $E$ is applied to move the polarons,

$$
H_{E}=|e| E \sum_{n, s}\left(n a+u_{n}\right)\left(C_{n, s}^{+} C_{n, s}-1\right)
$$

where $e$ is the electron charge and $a$ is the distance between the neighboring $\pi$ planes. In this case, each state $\psi_{\mu}$ is spin mixed and could be expressed on the basis of Wannier wave function,

$$
\psi_{\mu}=\left(\begin{array}{c}
\sum_{n} Z_{\mu, n, \uparrow}|n\rangle \\
\sum_{n} Z_{\mu, n, \downarrow}|n\rangle
\end{array}\right)
$$

where $Z_{\mu, n, s}$ is the probability amplitude of state $\psi_{\mu}$ with spin $s$ at site $n$. The evolution of the electronic states can be obtained by solving the time-dependent Schrödinger equation. The displacement $u_{n}$ is determined by the classical Newton equation,

$$
\operatorname{M} \ddot{u}_{n}(t)=2 \alpha\left[\rho_{n, n+1}(t)-\rho_{n-1, n}(t)\right]-K\left[2 u_{n}(t)-u_{n+1}(t)-u_{n-1}(t)\right]+|e| E\left[\rho_{n, n}\right.
$$

where the density matrix $\rho_{n, m}(t)=\sum_{\mu, s} Z_{\mu, n, s}^{*}(t) Z_{\mu, m, s}(t)$. Time-dependent Schrödinger equation and the Newton equation are solved by the Runge-Kutta method of order eight with step-size control ${ }^{8}$. To track the transmission of the polarons, we calculate the centre of the charge density $p_{c}{ }^{9}$,

$$
p_{c}=\left\{\begin{array}{lc}
\frac{N \theta}{2 \pi} & \left\langle\cos \theta_{n}\right\rangle \geq 0 \text { and }\left\langle\sin \theta_{n}\right\rangle \geq 0 \\
\frac{N(\pi+\theta)}{2 \pi} & \left\langle\cos \theta_{n}\right\rangle \geq 0 \\
\frac{N(2 \pi+\theta)}{2 \pi} & \text { otherwise }
\end{array}\right.
$$

where $N$ is the total number of monomers, $\left\langle\sin \theta_{n}\right\rangle=\sum_{n, s} Z_{N+1, n, s}^{*} Z_{N+1, n, s} \sin$ $(2 \pi n / N),\left\langle\cos \theta_{n}\right\rangle=\sum_{n, s} Z_{N+1, n, s}^{*} Z_{N+1, n, s} \cos (2 \pi n / N)$, and $\theta=\arctan \frac{\left\langle\sin \theta_{n}\right\rangle}{\left\langle\cos \theta_{n}\right\rangle}$. The velocity of the polaron at time $t$ is given by $v=p_{c}(t) / t$. 
Since the conductivity $\sigma$ could be calculated by $\sigma=q(n \uparrow \mu \uparrow+n \downarrow \mu \downarrow)$, where $q$ is the electron charge, $n$ is electron density, and $\mu$ is the charge mobility. $\uparrow$ and $\downarrow$ refer to spin up and spin down electrons. In addition, the mobility $\mu$ could be calculated by $\mu=v /$ $E$, where $v$ is the velocity of the electron and $E$ is the magnitude of applied electric field. In the model, our primary goal is to confirm the capability of helical PANIs to select specific electron spins. Therefore, the same amount of $n \uparrow$ and $n \downarrow$ were injected. Moreover, both $q$ and $E$ are the same for spin up and spin down electrons. Consequently, the only difference between spin up and spin down electrons is the velocity and this velocity difference was subsequently used to define the spin selectivity. The spin selectivity was qualitatively expressed by

$$
(v \uparrow-v \downarrow) /(v \uparrow+v \downarrow)
$$

where $v \uparrow$ and $v \downarrow$ are the velocity of spin up and spin down polarons respectively. Here, the parameters: $t_{0}=2.5 \mathrm{eV}, \alpha=4.1 \mathrm{eV} / \AA \mathrm{A}, \mathrm{K}=21 \mathrm{eV} / \AA^{2}, M=1349.14 \mathrm{eV} \cdot f \mathrm{~s}^{2} / \AA^{2}$, $a=1.22 \AA, t_{s o}=0.05 t_{0}, U=0.5 \mathrm{eV}, E=2.5 \times 10^{-4} V / \AA^{10-14}$. The twist and helix angle are calculated by measuring the helical pitch and diameter of a helical PANI microfiber where $\Delta \varphi \sim 0.03^{\circ}, \phi \sim 60^{\circ}$ are estimated (Figure S17 and Table S1). In addition, all of the parameters are summarized in Table S2. Moreover, by using reflection symmetry, the left-handed PANI helical microfiber is transformed into a right-handed conformation where the twist angle is flipped from $\Delta \varphi$ to $-\Delta \varphi$, and the helix angle is changed form $\phi$ to $\pi-\phi$. No changes have been made for other parameters. 

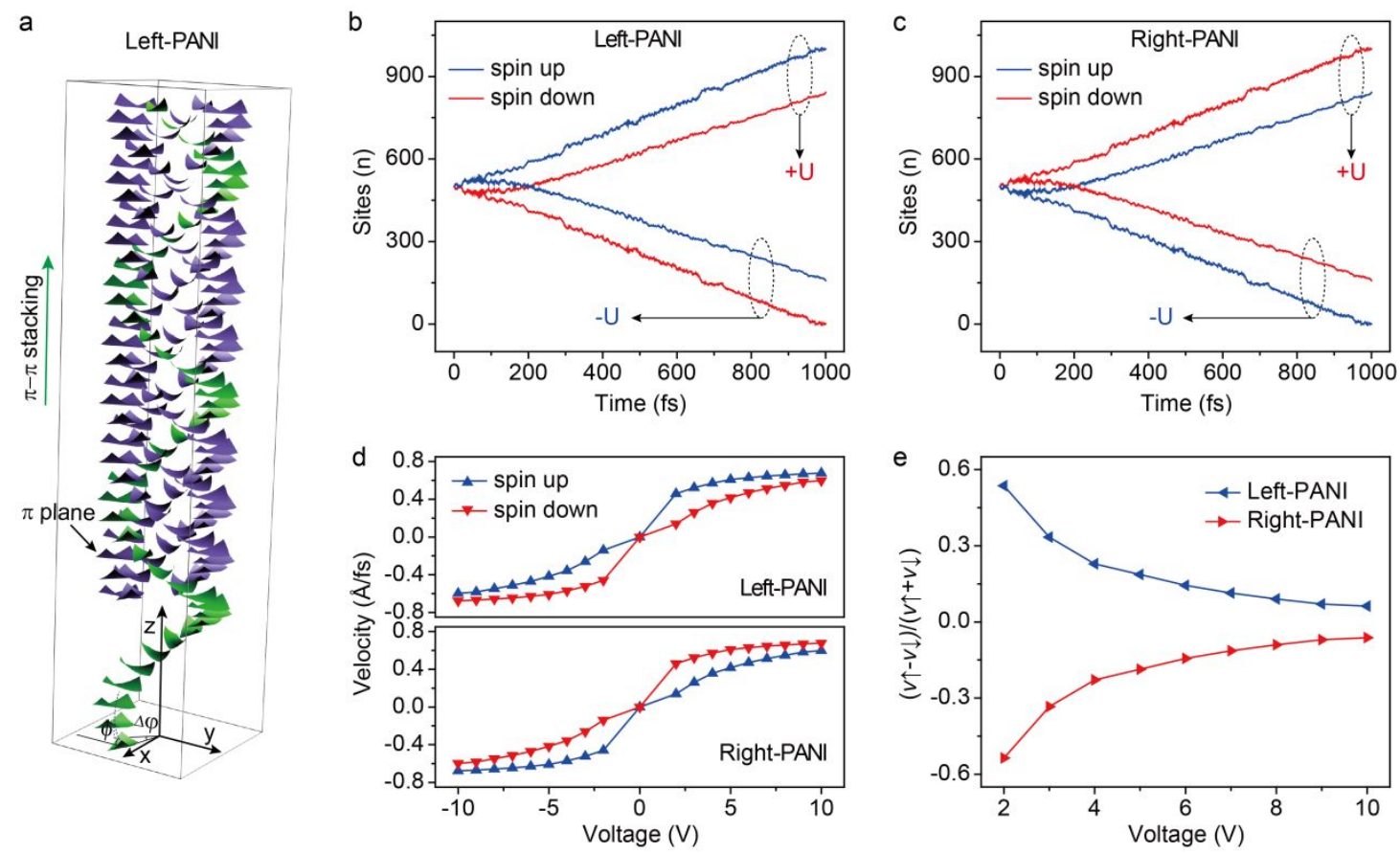

Figure S18. Simulation of spin selectivity in helical PANI microfibers. (a) The schematic illustration of a left-handed helical PANI microfiber based on structural characterizations (Figure S1). The $\pi$ planes of chiral PANI molecules arranged perpendicular to, while the $\pi-\pi$ stacking direction was along, the long axis of helical microfibers. The precise twist $(\Delta \varphi)$ and helix angle $(\phi)$ could hardly be acquired from the structural analysis. By carefully measuring the helical pitch and diameter of helical microfibers (Figure S17 and table S1), here, in this simulation, $\Delta \varphi=0.03^{\circ}, \phi=60^{\circ}$, and adjusting the angles within a reasonable range (not reverse the helical conformation) would not qualitatively flip the spin selective effect. (b) and (c) Time-dependent transmission of a polaron with opposite spins, namely spin up and spin down, in the helical potential of left- and right-handed PANI microfibers. (d) The transmission velocity of the polaron with spin up or spin down information as a function of placed bias in left- and right-handed helical PANI microfibers. (e) The spin selective capability as a function of placed bias for left- and right-handed helical PANI microfibers. 


\section{References}

1. Su, W. P.; Schrieffer, J.; Heeger, A. J., Solitons in Polyacetylene. Phys. Rev. Lett. 1979, 42,1698 .

2. Hohenadler, M., Interplay of Site and Bond Electron-Phonon Coupling in One Dimension. Phys. Rev. Lett. 2016, 117, 206404.

3. Rashba, E., Theory of Electrical Spin Injection: Tunnel Contacts as a Solution of the Conductivity Mismatch Problem. Phys. Rev. B 2000, 62, R16267.

4. Guo, A.; Sun, Q., Spin-Dependent Electron Transport in Protein-Like Single-Helical Molecules. Proc. Natl. Acad. Sci. U.S.A. 2014, 111 (32), 11658-11662.

5. Hubbard, J., Electron Correlations in Narrow Energy Bands. Proc. R. Soc. A 1963, 276, 238-257.

6. Vela, S.; Verot, M.; Fromager, E.; Robert, V., Electron Transport through a Spin Crossover Junction. Perspectives from a Wavefunction-Based Approach. J. Chem. Phys. 2017, 146, 064112.

7. Verot, M.; Borshch, S. A.; Robert, V., Spin Filtering in Molecular Junction: Magnetoresistance Evaluation from Wave-Function Calculations. J. Chem. Phys. 2013, 138, 094105.

8. An, Z.; Wu, C. Q.; Sun, X., Dynamics of Photogenerated Polarons in Conjugated Polymers. Phys. Rev. Lett. 2004, 93, 216407.

9. Wang, H.; Li, Y.; Li, D.-m.; Cui, B.; Liu, D.-S., Polaron Spin Filtering in an Organic Ferromagnetic Polymer: A Dynamics Simulation. Phys. Chem. Chem. Phys. 2016, 18, 503-509. 10. Sun, Z.; Stafström, S., Bipolaron Recombination in Conjugated Polymers. J. Chem. Phys. 2011, 135, 074902.

11. Meng, R.; Yin, S.; Zheng, Y.; Yang, L.; Xie, S.; Saxena, A., Phase-Breaking Effect on Polaron Transport in Organic Conjugated Polymers. Org. Electron. 2017, 49, 33-38.

12. Ribeiro, L. A.; da Cunha, W. F.; de Oliveira Neto, P. H.; Gargano, R.; e Silva, G. M., Dynamical Study of Impurity Effects on Bipolaron-Bipolaron and Bipolaron-Polaron Scattering in Conjugated Polymers. J. Phys. Chem. B 2013, 117, 11801-11811.

13. Di, B.; Meng, Y.; Wang, Y. D.; Liu, X. J.; An, Z., Electroluminescence Enhancement in Polymer Light-Emitting Diodes through Inelastic Scattering of Oppositely Charged Bipolarons. J. Phys. Chem. B 2011, 115, 9339-9344.

14. Guo, A.; Sun, Q., Spin-Selective Transport of Electrons in DNA Double Helix. Phys. Rev. Lett. 2012, 108, 218102. 